\title{
Preschool Teachers and Caregivers Positioning and Re Positioning in Preschool Due to New Division of Responsibilities in Policy Documents
}

\author{
Anna Öqvist' ${ }^{1}$, Sara Cervantes ${ }^{2}$ \\ Luleå University of Technology
}

\begin{abstract}
In Swedish preschools two occupational categories working side-by-side in working team: preschool teachers and caregivers. In 2011, a new educational reform was introduced in Sweden were preschool become a school form in its own right within the overall educational system. The Swedish preschool teacher profession was changed and strengthened were preschool teachers were given a clearer responsibility for the quality of the educational practice and for the work being carried out in line with the curriculum assignment. This resulted in a division of labor between the working team, the preschool teacher and the preschool principal. This study explores how preschool teacher and caregivers in Swedish preschools position themselves in relation to the changed responsibility between preschool teachers and the work team in preschool practice. Using a qualitative research design, this study collected data comprising 17 open-ended questionnaire with preschool teachers and caregivers in preschool. The result shows that both preschool teachers and caregivers position themselves as equal doing the same work, which indicate a challenge to reposition by moving away from their previous position with equal responsibility in the working team. Caregivers degrade preschool teachers' profession and position and upgrade their own competence and position as equal preschool teachers. Preschool teachers on the other hand upgrade caregivers' competence and position and degrade their own competence and position.
\end{abstract}

Keywords: Caregivers; Leadership; Positioning; Preschool; Preschool teacher 